\title{
Ignorance is not bliss: managing uncertainty in acute stroke treatment in the COVID-19 era
}

\author{
Aravind Ganesh $^{1} \cdot$ Johanna M. Ospel ${ }^{1,2} \cdot$ Julie Kromm ${ }^{1,3} \cdot$ Mayank Goyal $^{1,4}$ (D) \\ Received: 27 September 2020 / Accepted: 21 October 2020 / Published online: 31 October 2020 \\ (C) Springer-Verlag GmbH Germany, part of Springer Nature 2020
}

Decision-making in medical emergencies like acute stroke is fraught with potential pitfalls at the best of times. Whereas patients often prefer utilizing shared decision-making with detailed information [1], emergent decisions are based on a limited pool of immediately available information that may not reflect physicians', patients', and caregivers' preferences. The COVID-19 era has introduced additional challenges in this information flow. We use the example of acute ischemic strokea prototypical high-stakes, time-pressured emergency- to examine how information flow and availability have changed during the pandemic and their implications for emergent decisions.

In conventional settings, physicians decide about thrombolysis and endovascular therapy (EVT) for acute ischemic stroke based on available "known" clinical and imaging information like the patient's deficits or presence of a large vessel occlusion. This information is often provided by caregivers/ proxies, as the patients' deficits render them unable to cooperate or communicate [2]. Proxies base their decisions on how the physicians frame the patient's prognosis and the risks versus benefits of treatment. This interaction also builds a therapeutic alliance when facing uncertain treatment outcomes. Such discussions occur very rapidly (mean time of $2.7 \mathrm{~min}$ in one study) [2]. Several data points are variably available, depending on access to previous medical records and imaging

Mayank Goyal

mgoyal@ucalgary.ca

1 Calgary Stroke Program, Department of Clinical Neurosciences, Foothills Medical Centre, University of Calgary, 1403 29th St. NW, Calgary, AB T2N 2T9, Canada

2 Department of Radiology, University Hospital Basel, University of Basel, Basel, Switzerland

3 Department of Critical Care Medicine, University of Calgary, Calgary, Canada

4 Department of Radiology, Foothills Medical Centre, University of Calgary, 1403 29th St. NW, Calgary, AB T2N 2T9, Canada technology, like identifying a mismatch between the ischemic core and penumbra, differentiating old and new strokes, and knowledge about the patient having tortuous vessels or other potential technical challenges for EVT.

Numerous relevant factors that are potentially "knowable" are often lost to an "information bottleneck" (Fig. 1a) caused by time pressures or inter-personal factors, including how the patient, caregiver, and physician interpret the patient's quality of life; the proxy's true degree of "closeness" or investment in the patient's daily life (affecting their interpretation of the patient's functional status and wishes); the physician's experience with treating someone like the patient and their practice-specific incentives/disincentives for treatment; and the patient's current support system, their ability to pay for different options, and the patient's/proxy's understanding of the limits of therapy. In addition, there are other unknowable factors operating subconsciously behind a "knowledge barrier," including the physician's concerns about litigation, the physician's or proxy's personal philosophies (e.g., utilitarianism versus egalitarianism) or potential unconscious biases (e.g., ableism, ageism, sexism, racism), and the caregiver's willingness and bandwidth to care for the patient with their potential post-stroke disability [3]. Other factors behind the "knowledge barrier" can only be known in the future; information about them does not exist at the time of decision-making, including how the patient will actually fare with versus without treatment, the occurrence of unexpected treatment complications, the patient's response to rehabilitation, or their future resilience and support networks [4].

The COVID-19 pandemic has resulted in several systemic and workflow changes in emergency medicine - the added hassles of personal protective equipment (PPE) and COVID19 precautions, limited unit capacity and angiography suite availability due to healthcare system demands, and staff shortages and redeployments resulting in unfamiliar team compositions [5]. The risk of contracting COVID-19 has led to hospital avoidance behaviors among many patients with emergencies like stroke, whereas social-distancing restrictions 
a STANDARD SITUATION

\begin{tabular}{|c|c|c|c|}
\hline Physician Factors & Patient Factors & Caregiver Factors & \multirow{3}{*}{$\begin{array}{l}\text { 1. Need for personal } \\
\text { protective } \\
\text { equipment (PPE) } \\
\text { and precautions }\end{array}$} \\
\hline $\begin{array}{l}\text { - Unconscious biases } \\
\text { - Litigation concerns } \\
\text { Personal beliefs } \\
\text { regarding } \\
\text { treatment effect }\end{array}$ & $\begin{array}{l}\text { - Outcome with vs } \\
\text { without treatment } \\
\text { - Future resilience } \\
\text { - Future support } \\
\text { networks } \\
\text { - Post-stroke } \\
\text { complications }\end{array}$ & $\begin{array}{l}\text { - Bandwidth to care } \\
\text { for the patient } \\
\text { - Future willingness } \\
\text { to care for patient } \\
\text { - Future resilience } \\
\text { - Financial burden }\end{array}$ & \\
\hline \multicolumn{3}{|c|}{ Knowledge Barrier } & \\
\hline $\begin{array}{l}\text { - Workload, fatigue } \\
\text { - Treatment-based } \\
\text { incentives } \\
\text { - Perception of } \\
\text { patient's quality of } \\
\text { life (QoL) }\end{array}$ & $\begin{array}{l}\text { - Pre-stroke } \\
\text { functional status \& } \\
\text { medical history } \\
\text { - Personal wishes }\end{array}$ & $\begin{array}{l}\text { - "Closeness" to } \\
\text { patient } \\
\text { - Current willingness } \\
\text { to care for patient } \\
\text { - True view of } \\
\text { patient's QoL }\end{array}$ & \multirow{3}{*}{$\begin{array}{l}\text { 2.Social distancing } \\
\text { restrictions: no } \\
\text { caregiver or proxy } \\
\text { at bedside } \\
\text { 3.Limited unit } \\
\text { capacity, } \\
\text { angiography suite } \\
\text { availability } \\
\text { 4. Staff shortages } \\
\text { 5. Hospital avoidance }\end{array}$} \\
\hline \multicolumn{3}{|c|}{ Information Bottle-Neck } & \\
\hline $\begin{array}{l}\text { - Perception of } \\
\text { technical skills } \\
\text { - Stated prognosis } \\
\text { - Estimates of } \\
\text { treatment success } \\
\text { \& complications }\end{array}$ & $\begin{array}{l}\text { - Clinical deficits } \\
\text { - Age } \\
\text { - Vital parameters } \\
\text { - Imaging findings }\end{array}$ & $\begin{array}{l}\text { - Social/biological } \\
\text { relationship } \\
\text { - Stated role } \\
\text { - Stated view of } \\
\text { patient's pre- } \\
\text { stroke status }\end{array}$ & \\
\hline
\end{tabular}

Unknowable factors: information does not exist at the time of decision-making

Potentially knowable factors: information does exist but may be hard or impossible to obtain prior to decision-making

Knowable factors: information does exist and is easy to obtain prior to decision-making b COVID-19 SITUATION

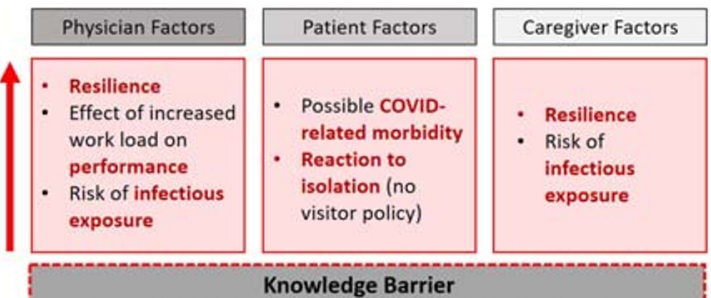

Factors above are impossible to obtain at the time of decision-making
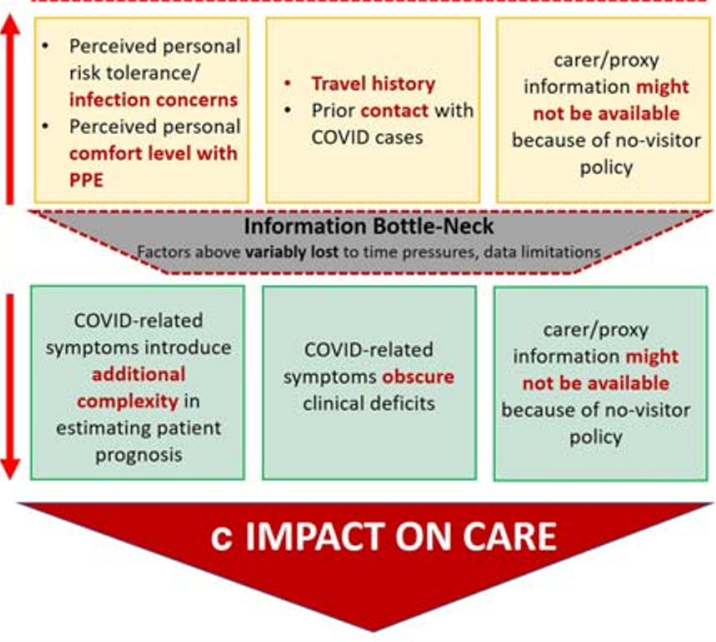

\section{d MITIGATING IMPACT ON CARE}

- Simulation training

- Greater utilization of telemedicine

- Local triage and transport coordination units

- Lower threshold for direct-to-mothership paradigms

- 'Post-mortem' analysis after every case

- Enhanced utilization of centralized records

Fig. 1 During typical emergency medical scenarios like ischemic stroke, there are various factors that are immediately available ("known") at the time of decision-making for physicians, patients, and their caregivers/ proxies, whereas other factors are potentially knowable but lost to an "information bottleneck" and still others lie hidden behind a "knowledge barrier" (a). The COVID-19 pandemic has resulted in a constellation of systemic and workflow changes that have resulted in additional unique

have resulted in no-visitor policies at many institutions, meaning there is often no caregiver/proxy at the bedside [6]. These changes further compromise and complicate information flow for acute treatment decisions, generally resulting in a greater information bottleneck and more factors trapped behind the knowledge barrier (Fig. 1b). Without readily available proxies, critical information like when the patient was last-seenwell, his/her pre-stroke functioning, comorbidities, advanced care directives, and COVID-specific information like travel/ contact exposures are easily lost to the information bottleneck. The physical examination may be limited by "protected" codes or clouded by the patient's need for respiratory support

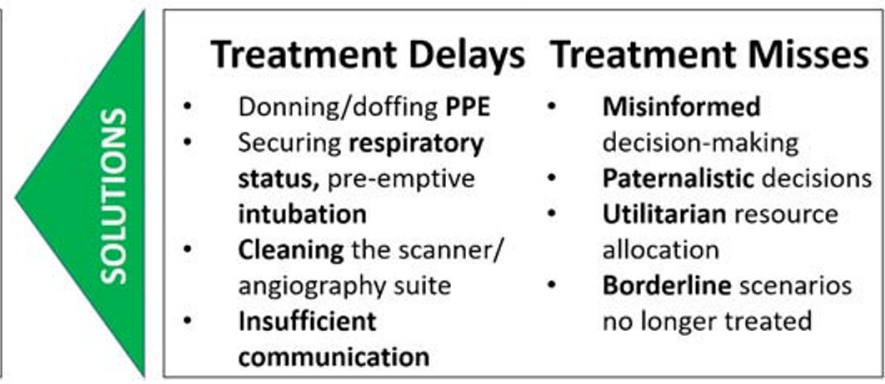

challenges in information flow for emergency medical decision-making (b). These challenges have a very tangible impact on patient care, largely in the form of treatment delays and missed opportunities for treatment (c). By recognizing the challenges to information flow and proactively responding to them, we may potentially mitigate this adverse impact on patient care (d)

with/without sedation for suspected COVID-19 [7]. Beyond the knowledge barrier lie numerous additional factors like the patient's unknown COVID-19 status and associated risk of complications, the physician's/team's risk tolerance about contracting COVID-19, and added uncertainty about treatment success posed by the unpredictable efficiency of new team members.

These added challenges in information availability can have a profound impact on patient care (Fig. 1c). Treatment delays may seem inevitable with competing priorities like donning/doffing PPE, greater attention to the patient's respiratory status with more frequent pre-emptive intubation, and a 
more obsessive need to clean the scanner and angiography suite after each case [8], with the team's speed often determined by its most risk-averse members. Communication with team members, the patient, and their proxy easily takes a back seat, particularly when it entails added efforts like finding and calling different phone numbers. There can be many missed opportunities for treatment, as reported by colleagues in China [6], with treatment decisions being easily misinformed by the added knowledge barriers and information bottlenecks. It becomes convenient to fall back on paternalistic and utilitarian models of decision-making, especially if treatments like EVT are viewed as being in competition with other uses of scarce resources during the pandemic [9].

Nevertheless, by appreciating the underlying pitfalls in information flow, we can adopt a more proactive and nuanced approach to emergency decision-making and potentially mitigate the impact of COVID-19 on patient care (Fig. 1d). First, we should vigorously train new and existing team members and practice COVID-19 workflows in simulations to streamline donning/doffing and cleaning procedures, thereby minimizing impediments to timely treatment [10]. Second, we should prioritize the use of telemedicine resources for efficient communication with caregivers/proxies to reduce our risk of misinformed decisions. Third, we should adapt our prehospital triage protocols for the COVID-19 era through both local triage and transport coordination units [11]. Recognizing the added risks of treatment delays or misses in these times, we may adopt a lower threshold for "direct-to-mothership" paradigms that bring stroke patients directly to comprehensive stroke centers to minimize blind spots. Fourth, the pandemic emphasizes the value of centralized electronic medical records to mitigate critical information loss, especially if a proxy is unreachable.

By responding to the inevitable barriers to information flow during the pandemic, we can ensure that our systems are best prepared to serve patients with acute stroke well beyond the COVID-19 era.

\section{Acknowledgements None}

Author contribution Aravind Ganesh: Conceptualization, drafting, and critical revision of the manuscript.

Remaining authors: Conceptualization and critical revision of the manuscript.

Funding Not applicable.

\section{Compliance with ethical standards}

Conflict of interest Dr. Ganesh reports membership in the editorial boards of Neurology, Neurology: Clinical Practice, and Stroke; speaker honoraria from NHS Health Education England and The Meritas Seminar
Series, Oxford; consulting fees from MD Analytics, MyMedicalPanel, Adkins Research Group, and Genome BC; research support from The Rhodes Trust, Wellcome Trust, the University of Calgary, Alberta Innovates, the Canadian Cardiovascular Society, and the Canadian Institutes of Health Research; and stock/stock options from SnapDx, TheRounds.ca, and Advanced Health Analytics (AHA Health Ltd), and has a provisional patent application (US 63/024,239) for a system to deliver remote ischemic conditioning or other cuff-based therapies. Dr. Ospel is supported by the Julia Bangerter Rhyner Foundation, University of Basel Research Foundation, and Freiwillige Akademische Gesellschaft Basel. Dr. Kromm has nothing to disclose. Dr. Goyal reports consulting fees from Medtronic, Stryker, Microvention, and Mentice, and has a patent for Systems of stroke diagnosis licensed to GE Healthcare.

Ethical approval Not applicable since this is an opinion piece and no patient data are contained in this manuscript.

Informed consent Not applicable since this is an opinion piece and no patient data are contained in this manuscript.

\section{References}

1. Rosenbaum JR, Bravata DM, Concato J, Brass LM, Kim N, Fried TR (2004) Informed consent for thrombolytic therapy for patients with acute ischemic stroke treated in routine clinical practice. Stroke 35(9):e353-e355. https://doi.org/10.1161/01.STR. 0000136555.28503 .55

2. Thomas L, Viswanathan A, Cochrane TI, Johnson J, O'Brien J, McMahon M, Santimauro JM, Schwamm LH (2012) Variability in the perception of informed consent for IV-tPA during telestroke consultation. Front Neurol 3:128. https://doi.org/10.3389/fneur. 2012.00128

3. Janz HL (2019) Ableism: the undiagnosed malady afflicting medicine. CMAJ 191(17):E478-E479. https://doi.org/10.1503/cmaj. 180903

4. Dhand A, Luke DA, Lang CE, Lee JM (2016) Social networks and neurological illness. Nat Rev Neurol 12(10):605-612. https://doi. org/10.1038/nrneurol.2016.119

5. Ospel JM, Goyal M (2020) Endovascular stroke treatment during the COVID-19 pandemic. Nat Rev Neurol. 16:351-352. https://doi. org/10.1038/s41582-020-0371-1

6. Zhao J, Li H, Kung D, Fisher M, Shen Y, Liu R (2020) Impact of the COVID-19 epidemic on stroke care and potential solutions. Stroke. 51:1996-2001. https://doi.org/10.1161/STROKEAHA. 120.030225

7. Khosravani H, Rajendram P, Notario L, Chapman MG, Menon BK (2020) Protected code stroke: hyperacute stroke management during the coronavirus disease 2019 (COVID-19) pandemic. Stroke 51:1891-1895. https://doi.org/10.1161/STROKEAHA.120. 029838

8. Nguyen TN, Abdalkader M, Jovin TG, Nogueira RG, Jadhav AP, Haussen DC, Hassan AE, Novakovic R, Sheth SA, OrtegaGutierrez S, Panagos PD, Cordina SM, Linfante I, Mansour OY, Malik AM, Narayanan S, Masoud HE, Chou SH, Khatri R, Janardhan V, Yavagal DR, Zaidat OO, Greer DM, Liebeskind DS (2020) Mechanical thrombectomy in the era of the COVID-19 pandemic: emergency preparedness for neuroscience teams: a guidance statement from the Society of Vascular and Interventional Neurology. Stroke 51:1896-1901. https://doi.org/10.1161/ STROKEAHA.120.030100 
9. Smith MS, Bonomo J, Knight WAt, Prestigiacomo CJ, Richards CT, Ramser E, Adeoye O, Bertsch S, Shirani P, Vagal A, Fichtenbaum CJ, Housholder A, Khatri P, Kleindorfer DO, Broderick JP, Grossman AW (2020) Endovascular therapy for patients with acute ischemic stroke during the COVID-19 pandemic: a proposed algorithm. Stroke 51:1902-1909. https://doi.org/10.1161/ STROKEAHA.120.029863

10. Kurz MW, Ospel JM, Kurz KD, Goyal M (2020) Improving stroke care in times of the COVID-19 pandemic through simulation: practice your protocols! Stroke 51:2273-2275. https://doi.org/10.1161/ STROKEAHA.120.030091
11. Goyal M, Ospel JM, Southerland AM, Wira C, Amin-Hanjani S, Fraser JF, Panagos P, AHA/ASA Stroke Council Science Subcommittees: Emergency Neurovascular Care (ENCC), the Telestroke and the Neurovascular Intervention Committees (2020) Prehospital triage of acute stroke patients during the COVID-19 pandemic. Stroke 51:2263-2267. https://doi.org/10.1161/ STROKEAHA.120.030340

Publisher's note Springer Nature remains neutral with regard to jurisdictional claims in published maps and institutional affiliations. 